\title{
ESTUDIO DE LAS COMUNIDADES DE INTERES PASCICOLA EN UN PUERTO DE MONTAÑA: I. ESTRUCTURA Y VALOR PASTORAL'
}

\author{
I. Alonso*; F. F. Bermúdez ; A. Garcia*; P. R. Revesado*; \\ A. R. MANTECÓN*; J.S. GONZÁlez $Z^{\star} \&$ G. CARLOS*
}

\begin{abstract}
RESUMEN.-En este trabajo se presenta un estudio de la estructura de las comunidades de pasto de montaña, como factor determinante del comportamiento ingestivo de los herbivoros. El mayor o menor impacto sobre estas comunidades depende de la selección que ejercen los animales, y ésta, a su vez, depende del valor que las plantas tienen para el herbivoro. El método aplicado en este caso para determinar este valor es el método "complex". Los resultados obtenidos se comparan con los de otros autores. La caracterización de las comunidades desde el punto de vista de su interés pascicola se completa con el estudio de los tipos biológicos basados en la clasificación de Raunkiaer.
\end{abstract}

ABSTRACT.-Studies of grazing plant communities in mountainous areas: 1. Structure and grass quality. The study of the structure of the mountainous grassland communities, as the main factor involved in the ingestive behaviour of herbivores, is presented in this work. The pressure on these communities depends on the selection excercised by the grazing animal and this, in turn, depends on the plant value or pastoral value for the herbivore. The "complex" method was used to determine this value. The results are compared with those of other authors. The "life forms" based on Raunkiaer's classification complete this study of the vegetal communities, defined from the point of view of their pastoral value.

RÉSUMÉ.- Étude des communautées de pâturage dans un port de montagne: I. Structure et valeur pastoral. Dans ce travail on présente l'étude de la structure des communautées de páturage de montagne comme un facteur déterminant du comportementingestive des herbivores. La grandeur de l'impact sur ces communautées dépend de la sélection faite par les animaux et, celle-ci en même temps, dépend de la valeur qu'ont les plantes pour l'herbivore. La méthode appliquée dans ce cas pour déterminer cette valeur est la méthode "complex". Les résultats obtenus sont comparées avec

1 Received, february 1992.

* Estación Agrícola Experimental de León (C.S.I.C.). Apartado 788, 24080 León, Spain.

** Departamento de Producción Animal, Universidad de León, 24071 León, Spain. 
ceux des autres auteurs. Sous le point de vue de leur intérêt pastorale, on complète la caractérisation des communautées avec l'étude des types biologiques basés sur la classification de Raunkiaer.

Key words: Mountainous grasslands, Communities, Structure, Pastoral value.

Las comunidades de pastos naturales de las áreas de montaña pertenecen a una gran variedad de tipos diferenciados, no sólo por su composición específica, sino por sus características estructurales. Estas diferencias estructurales, que pueden apreciarse claramente entre una comunidad de pasto y una comunidad de matorral, necesitan de estudios más detallados cuando se trata de comunidades fisionómicamente similares. La gran complejidad estructural y específica alcanzada en estas áreas de montaña se debe, por una parte a las condiciones ecológicas extremas, tanto climáticas (fuerte intensidad luminosa, heladas, sequía), como edáficas (falta de nutrientes); y por otra al intenso pastoreo a que han sido cometidas durante cientos de años. Aunque en la actualidad esta forma de aprovechamiento ha disminuido debido fundamentalmente a factores socioeconómicos, existe una creciente preocupación por el mantenimiento de estos sistemas pastorales que permitirian conservar unos paisajes milenarios de gran belleza y diversidad (GARCiA et al., 1989). Las características estructurales de los pastos constituyen además uno de los factores determinantes del comportamiento ingestivo de los hervíboros, con una importancia incluso mayor que la composición específica (Forbes, 1988; BARKMAN, 1989; ARIAS et al., 1990). La estructura del pasto varía en función de factores tales como el manejo y el grado de madurez alcanzado por la hierba. Se han señalado diferencias en la ingestión de pastos de la misma altura y disponibilidad que son atribuibles a diferencias estructurales que inciden en el tamaño de los bocados aprehendidos, componente del comportamiento ingestivo responsable, en última instancia, de la ingestión de hierba (HODGSON, 1985).

Los factores físicos, químicos y biológicos que condicionan la distribución de la vegetación actúan a diferentes escalas y pequeñas modificaciones en las condiciones del medio pueden determinar una variación paralela en la vegetación (DE NicolAs et al., 1980). El impacto de los herbívoros sobre el crecimiento de las plantas depende del tipo de tejido que seleccionen y del estado de desarrollo de las mismas. Este impacto es distinto según la especie de herbivoro, pudiendo existir diferencias incluso a nivel de razas. Sobre este apartado versa la segunda parte de este trabajo, en la que se analiza la intensidad de selección del pasto por dos razas ovinas españolas (REVESADO et al., 1991).

El estudio de las formas de vida basadas en la clasificación de Raunkiaer (ELLEMBERG, 1974), resultado tanto de la adaptación al medio climático como de los muchos siglos de intenso pastoreo, nos suministra una información 
complementaria. Las formas de crecimiento, más la estructura de la vegetación y su composición permiten entender cómo funcionan estas comunidades, ya que el éxito de los tipos biológicos depende de la combinación de sus características y una aproximación sólo analítica es insuficiente (WILLEMS, 1985; BARKMAN, 1988).

Además de la influencia que las características fisiognómicas tienen sobre los herbivoros, en la valoración de los pastos es necesario conocer la cantidad y la calidad de los mismos. Estos parámetros varian en función de la presión de pastoreo como respuesta a la ingestión del animal, pudiéndose apreciar variaciones en la producción de áreas pastadas respecto a las no pastadas. Los métodos más utilizados para estimar la ingestión en pastoreo se basan en la determinación de la excreción fecal (HODGSON \& RODRIGUEZ, 1971) y la determinación de la digestibilidad "in vitro" (TILLE \& TERRY, 1963) de la dieta seleccionada por animales provistos de fístulas esofágicas (VAN DYNE \& TORELL, 1964). La valoración de los pastos por estos métodos es muy compleja y en la segunda parte de este trabajo se presentan algunos de los resultados referentes a una de las comunidades estudiadas (REVESADO et al., 1991). En zonas muy diversas y extensas es necesario utilizar métodos de valoración alternativos, como los fitológicos conocidos con el nombre de "valor pastoral" de De Vries y Klapp, que modificados por DELPECH (1960 y 1976) Y DAGET \& POISSONET (1971), tienen una estrecha relación con el "valor nutritivo" del pasto (FERRER et al., 1976; AMELLA \& FERRER, 1977). SOSTARIC \& KOVACEVIC (1974) publicaron el método "complex" de valor pastoral, que tiene en cuenta la influencia de ciertos valores externos, como son: la intensidad de explotación, elementos nutritivos del suelo, proporción de gramíneas, presencia de substancias tóxicas, etc. En este trabajo se presenta el estudio de la estructura, diversidad, producción y valor pastoral de seis comunidades de interés pascicola. Los objetivos fueron: 1) conocer cómo los herbivoros han influido sobre las características funcionales y estructurales de los pastos con el tiempo y 2) comparar el efecto del pastoreo y eliminación del mismo, a corto plazo, sobre la estructura de la vegetación.

\section{Material y métodos}

Este estudio se llevó a cabo en una zona cercana al Puerto de San Isidro, en el norte de la provincia de León, entre 1400 y $1500 \mathrm{~m}$. de altitud. El Puerto ha sido pastado desde antiguo por rebaños de ovejas merinas trashumantes, así como ganado vacuno y caballar. El sustrato geológico de esta zona es variable alternando sustratos ácidos y básicos en los que se asientan diferentes tipos de pastos y matorrales. Se realizó un mapa de vegetación utilizando fotografía aérea (vuelo especial, CETFA, S. A., escala 1:8.000) que sirvió como base para estudios posteriores. Se identificaron nueve comunidades de las que se seleccionaron seis por su interés pascícola y por su extensión. Se instaló una jaula de exclusión de $1 \mathrm{~m}^{2}$ en cada una de ellas para 
determinar la producción y la estructura sin el efecto del pastoreo. La biomasa se estimó por el peso seco de la hierba cortada a $2 \mathrm{~cm}$ del suelo en esta superficie en el momento de máxima producción (principios de agosto).

En el año 1991 se realizaron un total de 120 inventarios, repartidos por igual entre todas las comunidades. Se usó un cuadrado de $0.5 \mathrm{~m}$. de lado. Los parámetros estimados fueron los porcentajes de cobertura de cada especie, cobertura total, porcentaje de suelo descubierto y porcentaje de líquenes y briófitos. También se midió en cada uno la altura media y la altura máxima del pasto. Con el porcentaje de cobertura de las especies se creó una matriz de 120 unidades de muestreo a la que se aplicó un análisis "cluster". Los grupos resultantes de este análisis se representaron esquemáticamente para mostrar la diferencia estructural entre las áreas pastadas y las zonas protegidas.

Se determinó el porcentaje de gramíneas, leguminosas y otras familias, y se estudió el porcentaje de tipos biológicos, siguiendo la clasificación de Raunkiaer. La diversidad se calculó por el índice de Shannon. Para determinar el valor pastoral se utilizó el método "complex" (SOSTARIC \& KovaceVIC, 1974) utilizando un programa desarrollado por uno de los autores, sin tener en cuenta Genista florida y G. obtusiramea pues su cobertura de $2 \mathrm{~m}$. del suelo fue aproximadamente de 95 a $100 \%$. La nomenclatura de las especies vegetales según Tutin et al. (1964-1980).

\section{Resultados}

La tabla 1 muestra los valores de producción, diversidad, número de especies por unidad de muestreo, porcentaje de leñosas y herbáceas, el valor pastoral y el indice de producción-calidad (producción $x$ índice de calidad) en $\mathrm{Tm} / \mathrm{Ha}$, de cada una de las comunidades determinadas por el

TABLA 1

Características de las comunidades estudiadas. (Characteristics of the studied communities).

\begin{tabular}{lrrrrrr}
\hline & N. stricta & C. vulgaris & E. arborea & G. florida & B. erectus & G. occidentalis \\
\hline Biomasa (Kg./Ha.) & 3.124 & 1.059 & 775 & 2.722 & 922 & 643 \\
Diversidad (Shannon) & 4.3 & 4.3 & 3.8 & 2.7 & 4.4 & 4.8 \\
N. $^{9}$ species/0.25 m & 11.8 & 18.6 & 12.6 & 7.3 & 20.6 & 19.3 \\
Especies leñosas (\%) & 0 & 11.3 & 17.6 & 9.7 & 2.0 & 7.7 \\
Especies herbáceas (\%) & 100.0 & 88.7 & 82.4 & 92.3 & 98.0 & 92.3 \\
Valor Pastoral & 13.40 & 26.42 & 22.88 & 7.79 & -22.78 & -17.71 \\
Indice Prod/cal & 41.86 & 27.98 & 17.73 & 21.20 & -21.00 & -11.39 \\
\hline
\end{tabular}

^Tm./Ha. de producción-calidad = producción $\mathrm{x}$ índice de calidad. 
análisis "cluster". Estas comunidades que se denominan con el nombre del matorral dominante, cuando está presente, están compuestas por especies herbáceas y un porcentaje más o menos variable de estrato arbustivo. Las comunidades con Nardus stricta, Calluna vulgaris, Erica arborea y Genista florida se distribuyen por los sustratos ácidos, mientras que las de Genista occidentalis y Bromus erectus ocupan los básicos.

En las figuras 1 a 6 se han presentado esquemáticamente las comunidades con y sin efecto de pastoreo, señalándose las especies con una presencia más significativa. En la figura 7 se indica el porcentaje que cada uno de los principales grupos: gramíneas, leguminosas y otras familias, aporta a cada comunidad.

En la tabla 2 se presenta la proporción de tipos biológicos en cada comunidad, según la clasificación de Raunkiaer. Los hemicriptófitos constituyen el porcentaje más elevado de especies en todas las comunidades con valores en torno al $70 \%$, repartiéndose el $30 \%$ restante entre caméfitos, geófitos y terófitos con valores muy similares. El pasto sobre caliza es la comunidad con más caméfitos herbáceos, cerca del $10 \%$, oscilando los valores en las otras comunidades en torno al $4 \%$. La proporción de los caméfitos leñosos en estas comunidades varía entre $5,8 \%$ en la comunidad de Calluna y el 9,4\% en la de Genista florida, con excepción del pasto de Nardus que carece de ellos. Los geófitos presentaron una variación mayor, desde $1,6 \%$ en la Comunidad de Genista occidentalis hasta el 13,1 \% en la de Nardus. Entre ellos podemos destacar por su importancia el Crocus nudiflorus y el Narcissus asturiensis que está presente en todas las comunidades. Los terófitos presentaron valores más elevados en las comunidades que crecen sobre sustrato calizo (Bromus erectus y Genista occidentalis).

TABLA 2

Porcentaje de tipos biológicos basados en la clasificación de Raunkiaer (TOTAL = número de especies; CAM.I = caméfitos leñosos; CAM.h = caméfitos herbáceos; HEMICR. = hemicriptófitos; GEOF. = geófitos; TEROF. = terófitos. (Percentage of lieforms following Raunkiaer's classification (CAM., = woody chamaephytes; HEMICR. = hemicryptophytes; GEOF. = geophytes; TEROF. = therophytes).

\begin{tabular}{lrrrrrr}
\hline & TOTAL & CAM., & CAM. & HEMICR. & GEOF. & TEROF. \\
\hline Nardus stricta & 23 & 0 & 3.5 & 82.7 & 10.3 & 3.5 \\
Calluna vulgaris & 52 & 6.1 & 8.2 & 73.5 & 6.1 & 6.1 \\
Erica arborea & 45 & 9.1 & 6.8 & 70.4 & 9.1 & 4.6 \\
Genista florida & 32 & 10.3 & 3.5 & 65.5 & 13.8 & 6.9 \\
Bromus erectus & 44 & 6.7 & 20.0 & 53.3 & 8.9 & 11.1 \\
Genista occidentalis & 62 & 8.1 & 11.3 & 69.3 & 1.6 & 9.7 \\
\hline
\end{tabular}


PIRINEOS 141-142

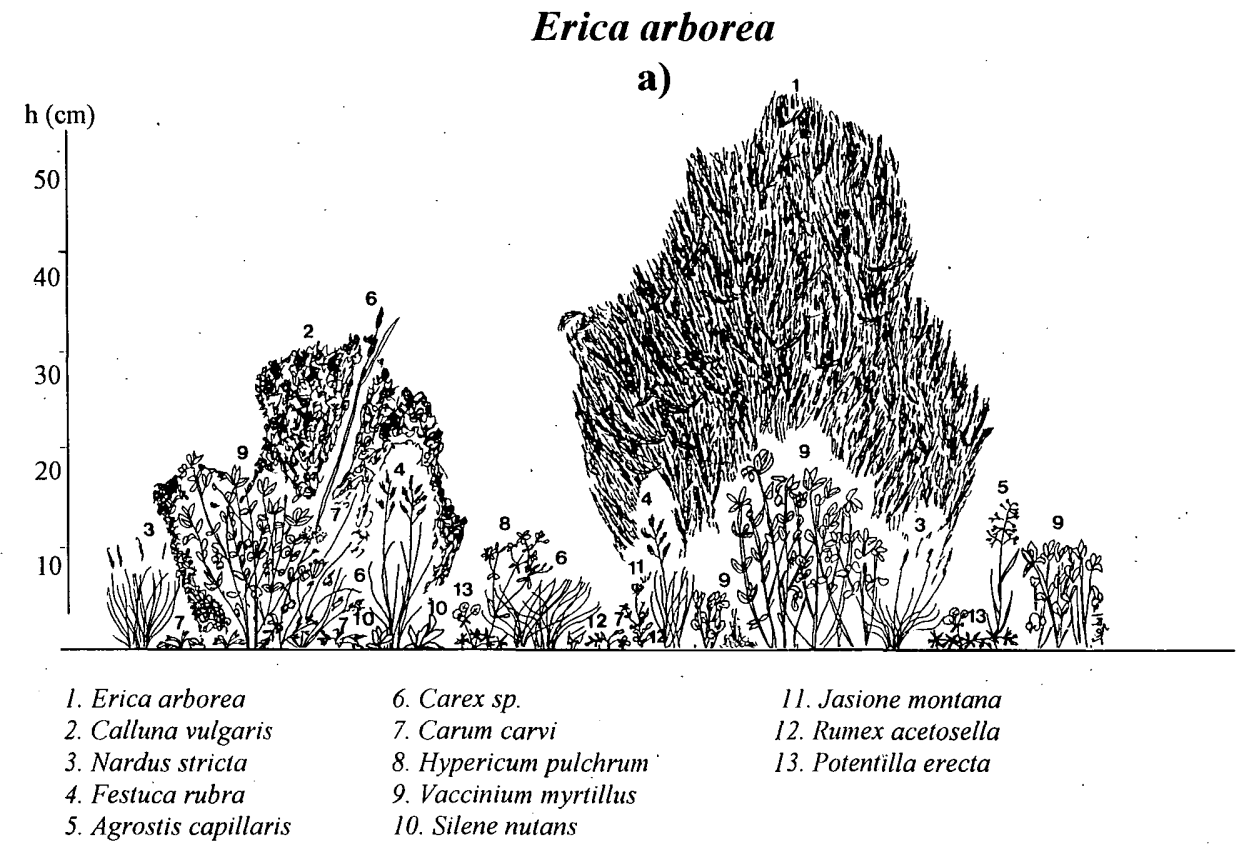

\section{Erica arborea}

b)

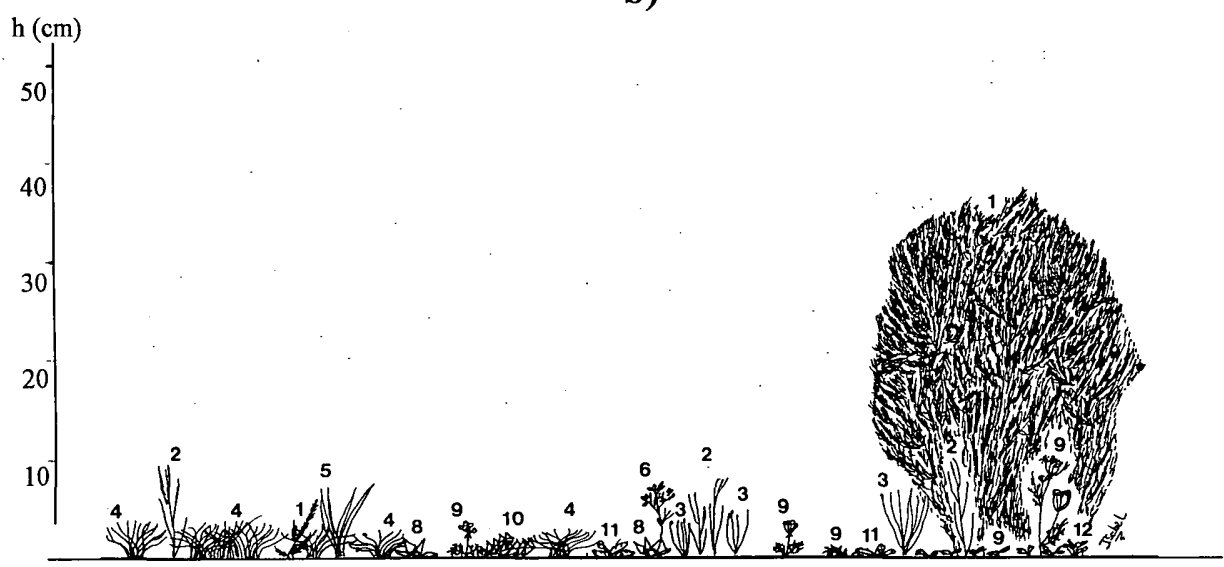
1. Erica arborea
5. Avenula sulcata
9. Taraxacum officinale
2. Agrostis capillaris
6. Genista obtusiramea
10. Hieracium pilosella
3. Festuca rubra
7. Rumex acetosella
11. Jasione montana
4. Nardus stricta
8. Carum carvi

Fig. 1. Estructura de la comúnidad de Erica arborea: a) sin pastar; b) pastada. (Erica arborea community structure: a) ungrazed; b) grazed).

8 
COMUNIDADES DE INTERES PASCICOLA

\section{Calluna vulgaris}

a)

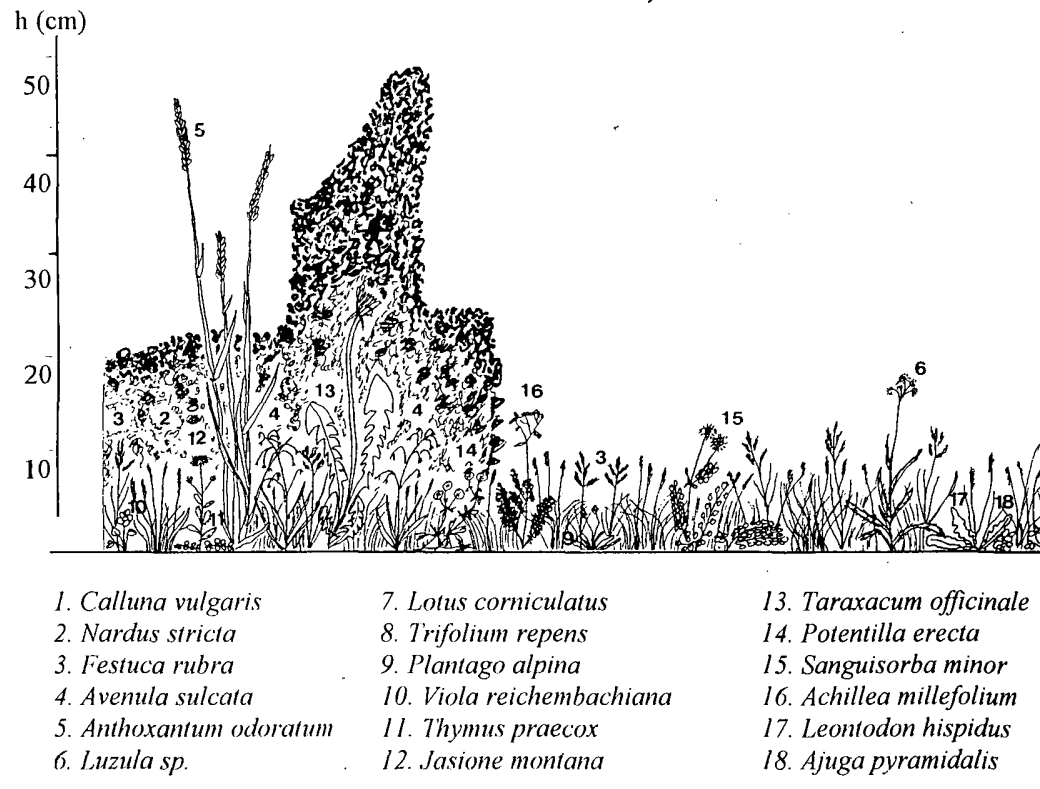

\section{Calluna vulgaris}

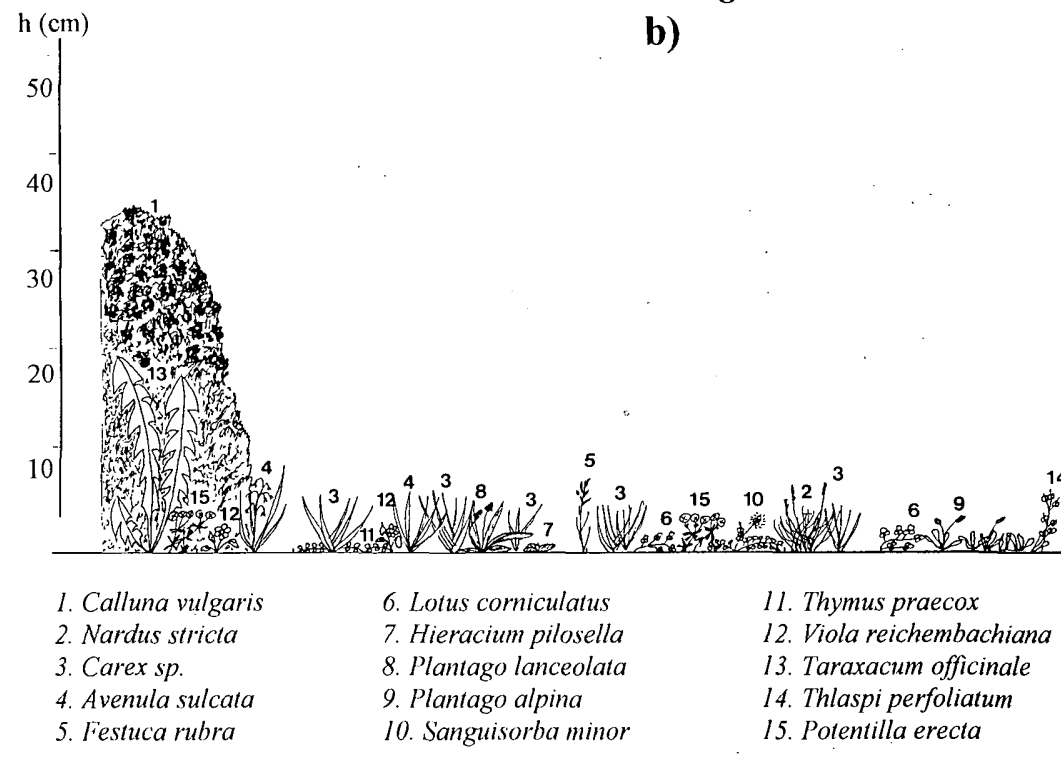

Fig. 2. Estructura de la comunidad de Calluna vulgaris: a) sin pastar; b) pastada. (Calluna vulgaris community structure: a) ungrazed; b) grazed). 


\section{Genista florida}

a)

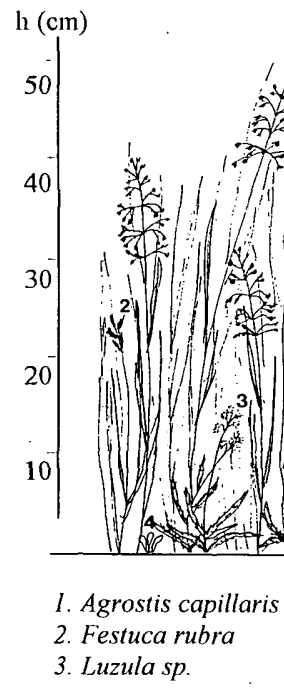

2. Festuca rubra
3. Luzula sp.

4. Rumex acetosella
5. Narcisus asturiensis
6. Conopodium majus

檽

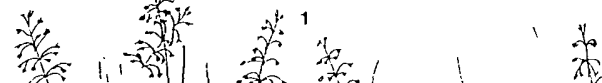

14

.

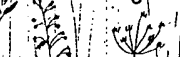

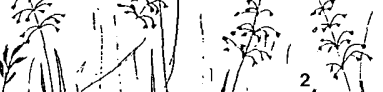
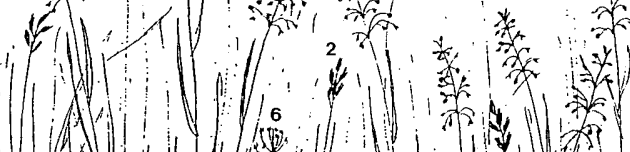

un

\section{Genista florida}

b)

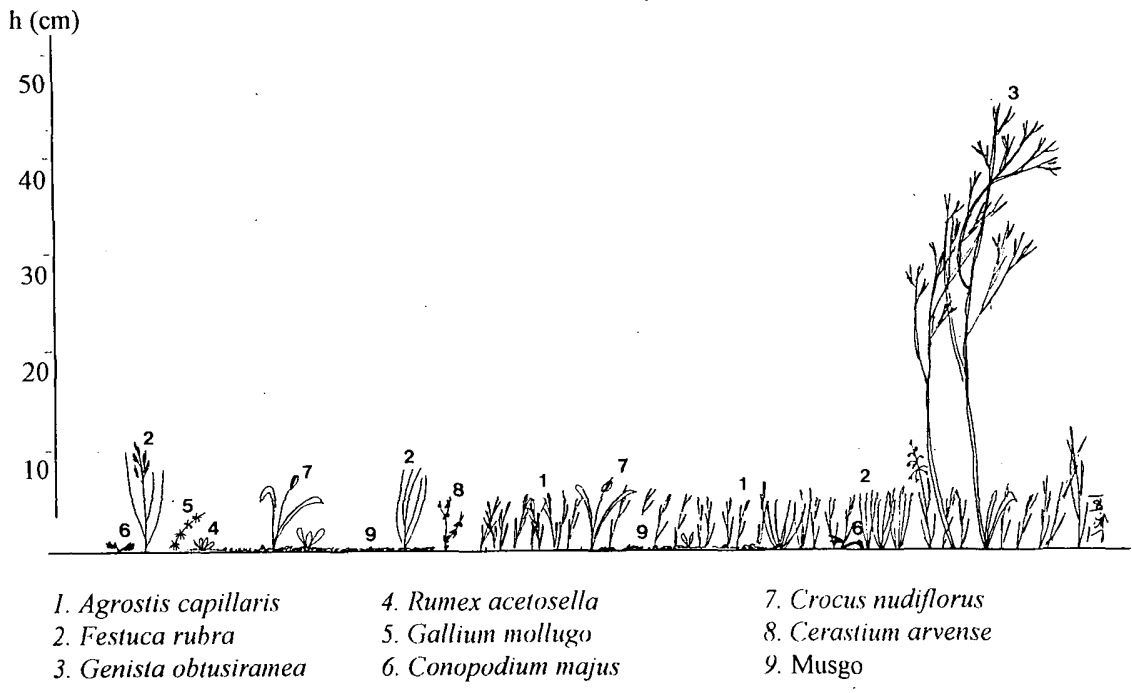

Fig. 3. Estructura de la comunidad de Genista florida: a) sin pastar; b) pastada. (Genista florida community structure: a) ungrazed; b) grazed).

10 
COMUNIDADES DE INTERES PASCICOLA

\section{Nardus stricta}

a)

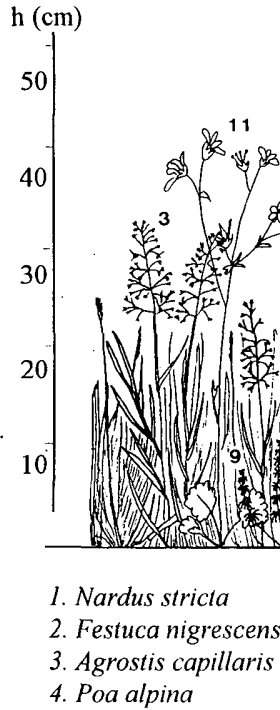

4. Poa alpina
5. Luzula sp.

6. Trifolium repens

7. Carum carvi

8. Ranunculus bulbosus
9. Galium verum

10. Plantago lanceolata

11. Saxifraga granulata

12. Achillea millefolium

Nardus stricta

b)

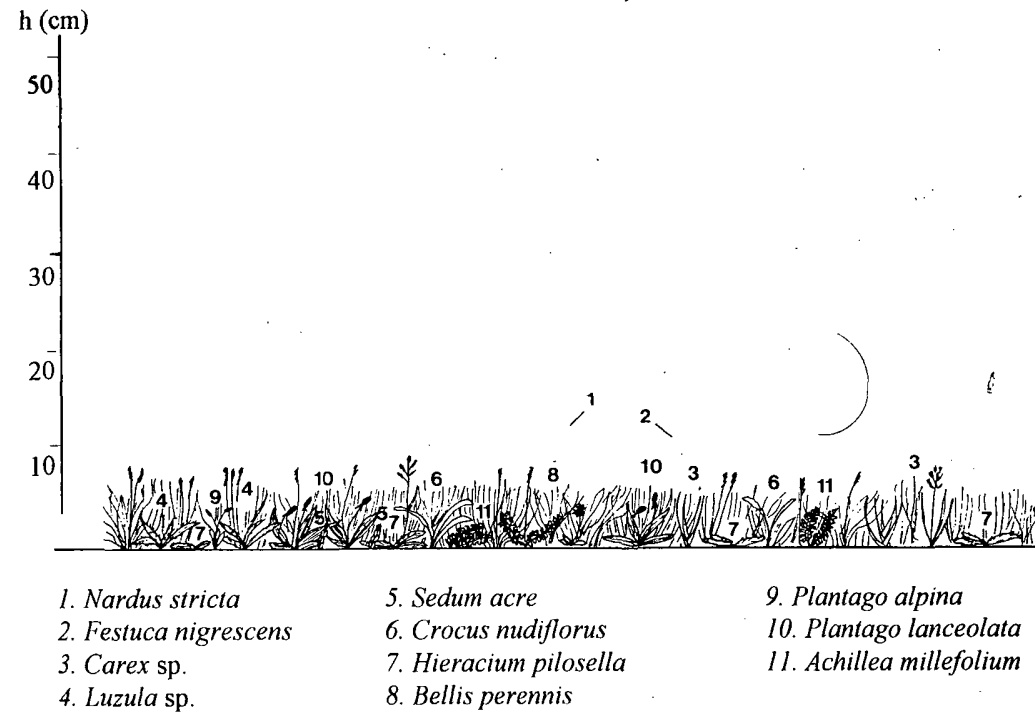

Fig. 4. Estructura de la comunidad de Nardus stricta: a) sin pastar; b) pastada. (Nardus stricta community structure: a) ungrazed; b) grazed). 
PIRINEOS $141-142$

\section{Genista occidentalis}

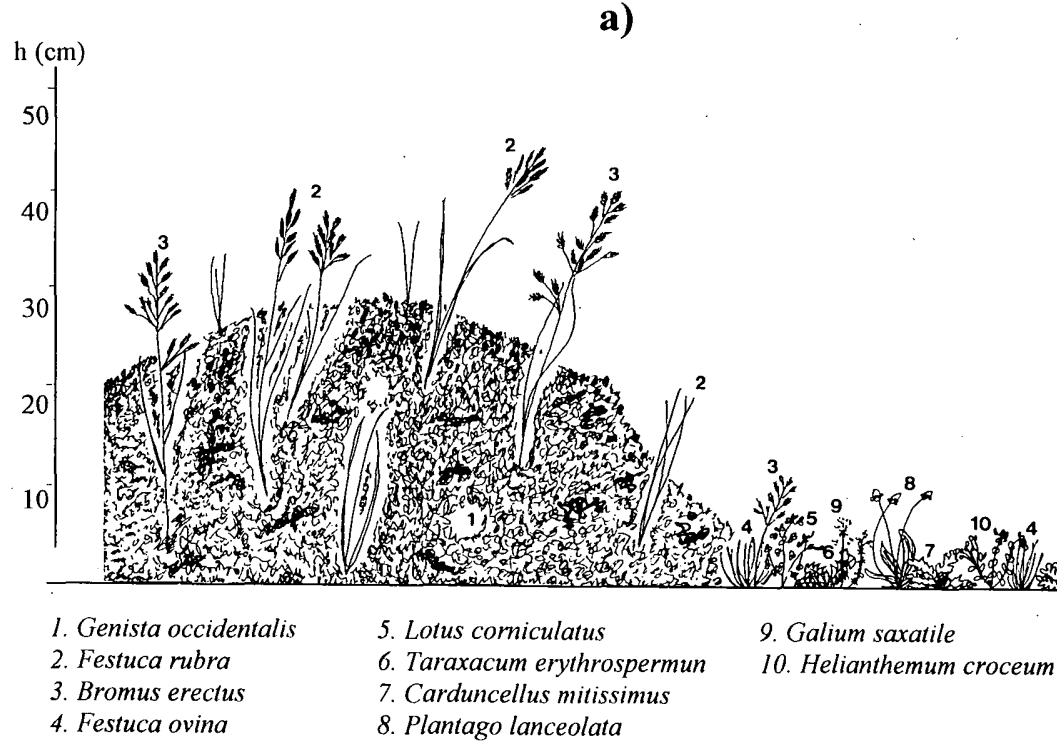

\section{Genista occidentalis}

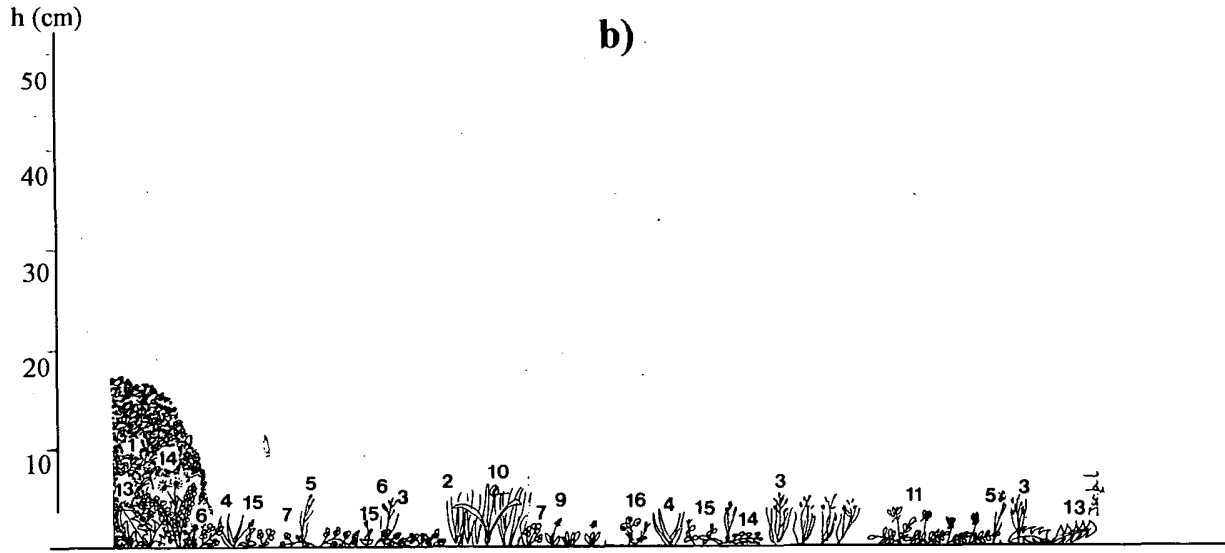

$\begin{array}{lll}\text { 1. Genista occidentalis } & \text { 7. Trifolium repens } & \text { 13. Taraxacum erythrospermun } \\ \text { 2. Festuca rubra } & \text { 8. Plantago lanceolata } & \text { 14. Sanguisorba minor } \\ \text { 3. Bromus erectus } & \text { 9. Plantago alpina } & \text { 15. Acinos alpinus } \\ \text { 4. Carex sp. } & \text { 10. Crocus nudiflorus } & \text { 16. Potentilla crantzii } \\ \text { 5. Agrostis capillaris } & \text { 11. Anthyllis vulneraria } & \\ \text { 6. Lotus corniculatus } & \text { 12. Bellis perennis } & \end{array}$

Fig. 5. Estructura de la comunidad de Genista occidentalis: a) sin pastar; b) pastada. (Genista occidentalis community structure: a) ungrazed; b) grazed).

12 
COMUNIDADES DE INTERES PASCICOLA

\section{Bromus erectus}

a)

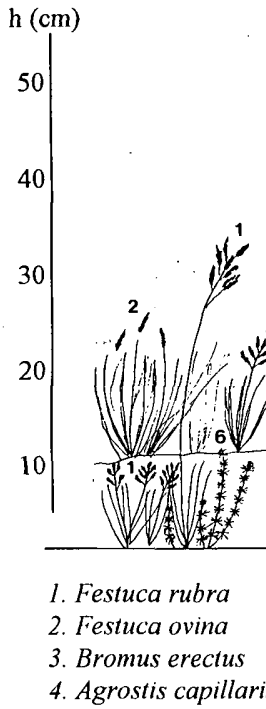

4. Agrostis capillaris

6. Galium verum

7. Achillea millefolium

8. Allium sp.

\section{Bromus erectus}

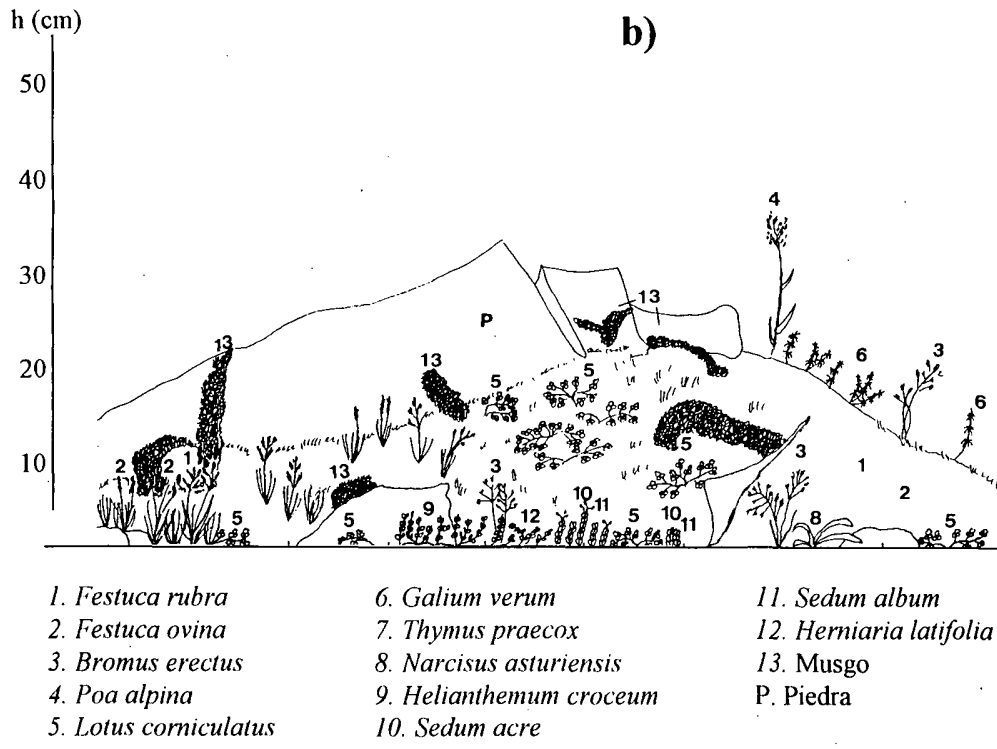

Fig. 6. Estructura de la comunidad de Bromus erectus: a) sin pastar; b) pastada. (Bromus erectus community structure: a) ungrazed; b) grazed). 


\section{Discusión}

El pastoreo continuado a lo largo del tiempo puede afectar a la distribución horizontal de las plantas dando como resultado comunidades con formas de crecimiento postradas y de pequeña altura, formando un césped continuo más o menos homogéneo, cuya descripción a nivel de comunidad debe basarse en la fisiognomia (MCNAUGHTON, 1984; MILCHUNAS \& LAUENROTH, 1989). En algunos de estos ecosistemas la fisiognomla actual es el resultado, tanto de un cambio en la composición específica, en el que las especies de porte alto son sustituidas por otras más bajas, como de la selección de formas de crecimiento rastreras. Una de las causas de estos cambios es la presión de pastoreo (MCNAUGHTON, 1983 y 1984), que parece ser es lo que sucede en las comunidades que nos ocupan. Fisiognómicamente se trata de comunidades de pequeña altura y con abundancia de formas de crecimiento adaptadas a esta presión, ya que han sido pastadas desde muy antiguo.

La altura del pasto es uno de los parámetros que más claramente caracteriza el cambio en la fisiognomía de la vegetación al comparar áreas protegidas o pastadas. El estrato herbáceo en las primeras tiene una media entre 12 y $18 \mathrm{~cm}$. Algunos individuos sobresalen por encima de este valor incluso triplicándolo. La comunidad pascicola con Genista florida y G. obtusiramea, por encontrarse en suelos más ricos, es la más alta cuando no es pastada, con una media de unos $30 \mathrm{~cm}$. e individuos aislados que sobrepasan los $50 \mathrm{~cm}$. El pasto con Genista occidentalis, especie que se distribuye formando almohadillas espinosas, ocupa zonas con pendientes elevadas situadas sobre sustrato calizo. Las alturas de las herbáceas en esta comunidad, varian mucho si están entre el matorral o al descubierto. En el primer caso pueden alcanzar los $40 \mathrm{~cm}$ y en el segundo no suelen sobrepasar los $10 \mathrm{~cm}$. Cuando es pastada, el aspecto de esta comunidad varía mucho adquiriendo el suelo un aspecto esquelético, en el que las plantas raramente miden más de $5 \mathrm{~cm}$.

En general, al ser pastadas, estas comunidades adquieren un aspecto más homogéneo. Las alturas no suelen superar los $7 \mathrm{~cm}$., aunque en los pastos de Bromion, donde la roca aflora frecuentemente, no sobrepasan los $203 \mathrm{~cm}$. La defoliación altera el balance de agua de toda la comunidad, reduciendo el número de hojas que transpiran y exponiendo áreas de suelo a la desecadora influencia del sol y viento, efecto que también fue apreciado por CRAWLEY (1983 y 1986). Sólo la comunidad con Nardus stricta mantiene el $100 \%$ de cobertura vegetal.

La producción está directamente relacionada con la altura. En las comunidades estudiadas varía entre $7775 \mathrm{Kg} / \mathrm{Ha}$ en la de Erica arborea y 3.124 $\mathrm{Kg} / \mathrm{Ha}$ en la de Nardus stricta. Esta última cantidad, junto con su valor pastoral, tiene en cambio una producción menor, con lo que el índice de producción-calidad disminuye respecto a la comunidad anterior. Las variables ambientales no pueden ser incluidas en la evaluación general de la 
calidad del pasto y fueron corregidas según el modelo propuesto por SOSTARIC \& KOVACEVIC (1974). Estas correcciones varian según la altitud, la exposición, la diversidad, el manejo... Al corregir el valor pastoral en función de estas variables ambientales hubo un aumento del valor en relación con la altitud y el número de especies beneficiosas, y una disminución del mismo relacionado con el tipo de manejo y en algunos casos por efecto de la sombra. Los valores obtenidos después de estas correcciones son menores que los de comunidades de prados (CHOCARRO et al., 1987 y NAVASCUES, 1987) pero se asemejan al de los pastos de las "rañas" en la región central (GARcia et al., 1988), no obstante, puesto que la producción en las "rañas" es mucho menor, el índice de producción-calidad es considerablemente mayor que en los pastos de las "rañas".

La diversidad es relativamente alta presentando valores de indice de Shannon H' próximos a 4. Estos valores son comparables a los encontrados por CHOCARRO et al. (1987) y GARCIA (1992) para comunidades de prados. La comunidad con Genista florida, sin embargo, tiene un valor de H' de 2,7 y sólo aparecen 7,3 especies como media en los inventarios alli realizados.

De los tipos biológicos según la clasificación de Raunkiaer (figura 7 y tabla 2), los hemicriptófitos constituyen en todas estas comunidades el tipo que más especies engloba, lo cual es característico de zonas de montaña, siendo las comunidades de Nardus-Festuca y Calluna las que tienen unas proporciones más elevadas. Estas zonas, debido a la altitud, tienen una atmósfera rarificada, que no amortigua los cambios de temperatura. Se dan por ello grandes contrastes ambientales en cortas distancias y temporalmente, a lo largo del dia o de las estaciones.

Los herbivoros pueden reducir, mantener o mejorar sus recursos alterando la arquitectura de la planta (MOPPER et al., 1991). En las comunidades objeto de estudio el efecto observado es una disminución de la oferta. Debido a la plasticidad fenotípica que muestran las especies, cuando se liberan de la presión del pastoreo la mayoria recuperan un porte alto. Reciprocamente la ingestión de los herbívoros es sensible a variaciones en la cantidad y altura de la hierba (HODGSON, 1981). Las diferencias de uso entre las comunidades se deben a su composición específica y a su mayor o menor complejidad vertical, y el efecto de los herbívoros sobre la biomasa es variable según el sistema de que se trate. AsI MCNAUGHTON.(1984) indica que, en el área de Serengueti, la biomasa de las gramíneas altas aumenta con el pastoreo, mientras que McGUINLEY \& WITHAM (1985), observaron que la biomasa de algunas plantas disminuye al ser pastadas y pueden permanecer en estado juvenil permanente por suprimirse su reproducción sexual.

Agradecimientos. Este trabajo ha sido realizado con la ayuda de los proyectos PB87-0349 y PB89-0039 financiados por la D.G.I.C.Y.T. 


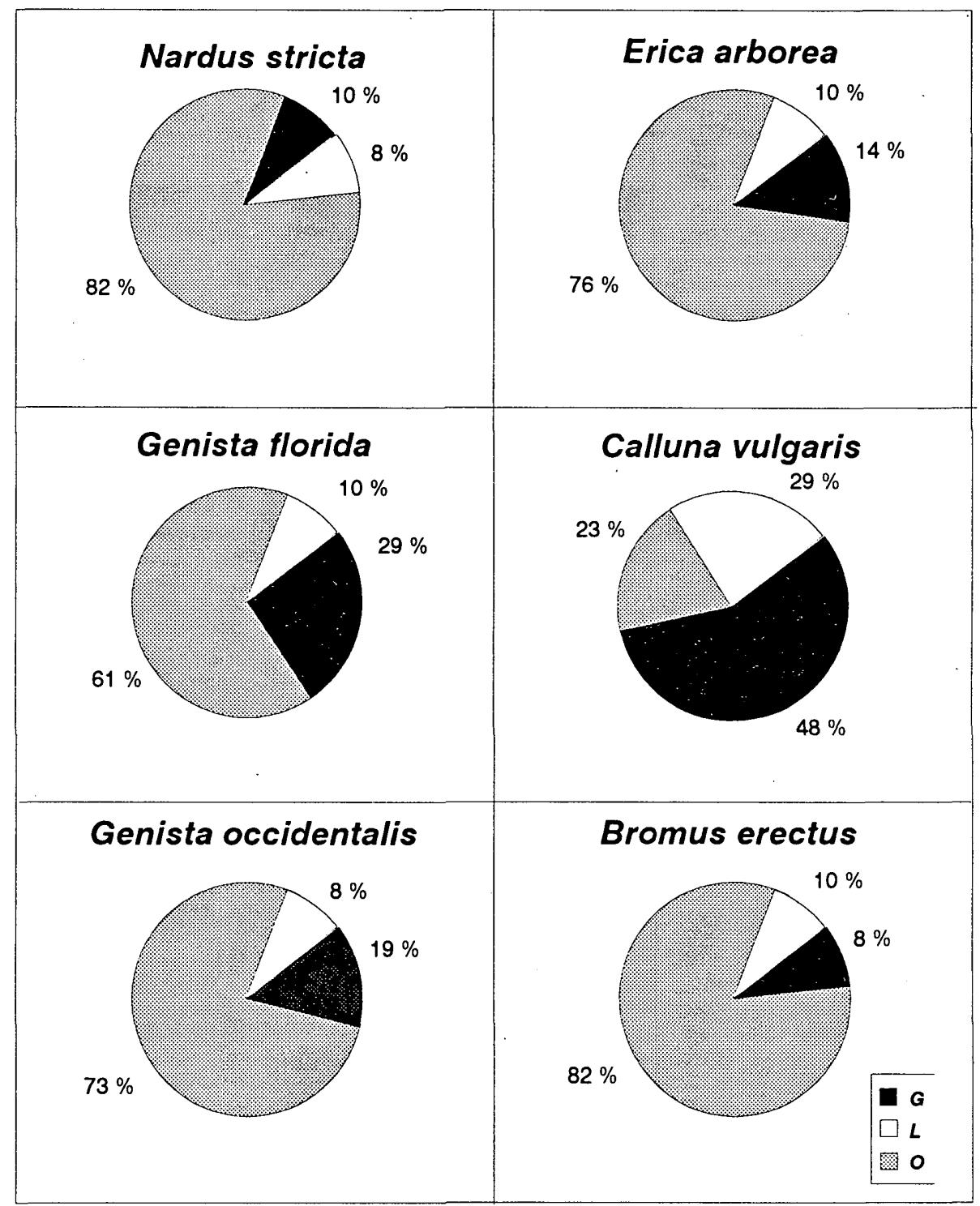

Fig. 7. Porcentaje de gramíneas, leguminosas y otras familias en las comunidades presentes. (Percentage of grasses, legumes and other families in the present communities). 


\section{Referenolas}

Amella, A. \& Ferrer, C. (1977): Utilización de un método fitológico en la determinación del valor nutritivo de pastos. Pastos, 7: 270-277.

ArIAs, J. E., DOUGHerTy, C., BRADLEY, N., Cornelius, P. L. \& LAURIAULT, L. M. (1990): Structure of tall fescue swards and intake of grazing cattle. Agronomy Journal, 82(3): 545-548.

BARKMAN, J. J. (1989): A tentative typology of european scrub and forest communities, based on vegetation texture and structure. En: Forests of the World: Diversity And Dynamics (Abstracts). Sjögren, E. (Ed:). Almqvist \& Wiksell International, Stockhoim, Sweden Uppsala.

BARKMAN, J. J. (1988): New systems of plant growth forms and phenological plant types. En: Plant Form and Vegetation Structure: adaptation, plasticity and relation to herbivory. Werger, M. J. A., van der Aart, P. J. M., During, H. J. y Verhoeven, J. T. A. (eds.). pp. 9-44. SPB Academic Publ., The Hague.

Chocarro gómez, C., fillat estaque, f., Garcia Ciudad, A. \& Miranda Pérez, P. (1987) Prados del Pirineo Central: composición florística y calidad. Pirineos, 129: 7-33.

Crawley, M. J. (1983): Herbivory: The Dynamics of Animal-Plant Interactions. Crawley, M. J. (Ed.). MBlackwell Scientific Publications. London.

Crawley, M. J. (1986): Plant Ecology. Crawley, M. J.(Ed.). Blackwell Scientific Publications. London. pp. 1-50.

DAGET, Ph. y POISSONET, J. (1971): Une méthode d'analyse phytologique des prairies. Critéres d'application. Ann. Argr. France, 22: 5-41.

DELPECH, R. (1960): Critères de jugement de la valeur agronomique des prairies. Fourrages, 4: 83-98.

DELPECH, R. (1976): Essais d'appréciation de la valeur agronomique à partir des donnés phytosociologiques. Coll. Phytos. V. Les Prairies Inondables. pp. 131-135. Lille. Vaduz.

De Nicolas, J. P., Fernández Galiano, E. \& Garcia quintas, M. A. (1980): Análisis de la microestructura espacial de la vegetación de los pastizales. Studia Oecologica, I: 109-130.

Ellembera, H. (1974): Zeigerwerte der GefäBpflanzen Mitteleuropas. Scripta Geobotanica IX.

FerRer, C., Amella, A. \& MAestro, M. (1976): Relación entre la composición florística y la ecología de pastos estivales pirenaicos, con su valor nutritivo. Trab. Instituto de Economia y Producciones Ganaderas del Ebro, 24: 1-26.

FORBES, T. D. A. (1988): Researching the plant-animal interface: the investigation of ingestive behaviour in grazing animals. J. Anim. Sci. 66: 2.369-2.379

Garcia, A., PAstor, J. \& Bermudez, F. F. (1988): Valor de los pastos en zonas degradadas de la región central. Avances en Alimentación y Mejora Animal, 28(2): 57-61.

Garcia, A., Revuelta, J., Bermudez, F. F. \& Mantecon, A. R. (1989): Conservación de los paisajes pastorales y politica agraria. II Jornadas sobre el paisaje, Segovia.

GARCiA, A. (1992): Conserving the species rich meadows of Europe. Agriculture Ecosystems and Environment, 40: 219-232.

HODGSON, J. \& RODRigueZ, J. M. (1971): The measurement of herbage intake in grazing studies. Ann. Rep. Grassland. Res. Inst., 132-140.

HoDGsoN, J. (1981): Variations in the surface characteristics of the sward and the shortterm rate of herbage intake by calves and lambs. Grass and Forage Science, 36: 49-57. 
Hodgson, J. (1985): Grazing and its influence on hill vegetation. En: Vegetation Management in Northern Britain. British Crop Production Council. Monografia n. ${ }^{2}$ 30, pp. 21-31. U. K.

McGinLeY, M. A. \& WITHAM, T. G. (1985): Central place foraging by beavers (Castor canadensis): a test of foraging predictions and the impact of selective feeding on the growth form of cottonwoods (Populus fremonti). Oecologia, 66: 558-562.

McNaughton, S. J. (1983): Serengeti grassland ecology: the role of composite environmental factors and contingency in community organization. Ecol. Monogr., 53: 291-320.

MCNAUGHTON, S. J. (1984): Grazing lawns: animals in herds, plant form and coevolution. Am. Nat., 124: 863-886.

MilchunAs, D. G. \& LAUENROTH, W. K. (1989): Three-dimensional distribution of plant biomass in relation to grazing and topography in the shortgrass steppe. Oikos, 55 : 82-86. Copenhagen.

MOPPER, S., MASCHINSKI, K., COBB, N. \& WITHAM, T. G. (1991): A new look at habitat structure: consequences of herbivore-modified plant architecture. En: Habitat Structure: The Physical Arrangement of Objects in Space. Bell, S. S., McCoy, E. D. y Mushinsky, H. R. (Eds). Suffolk. pp. 260-280. Chapman and Hall.

NAVAscues, I. (1987): Estructura y Valoración Agronómica de los Prados de Siega de la Comarca de Riaño, León. Tesis Doctoral. Facultad de Biología, Universidad de León.

Revesado, P. R., Mantecón, A. R., Gonzalez, J. S., Frutos, P., Ramos, G., Alonso, I., Garcia A. \& Bermudez, F. F. (1991): Estudio de las comunidades de interés pascícola en un puerto de montaña: Il evolución en la intensidad de selección del pasto por dos razas ovinas (Churra y merina). III Jornadas de Ecologla Terrestre, León.

Sostafic, K. \& KovaceVIC, J. (1974): Kompleksna Metoda Za Utvrdjvanje Kvalitete I Sumame Vrijednosti Travnjaka / Djetista. Universitatis Zagrabiensis. Facultatis Agronomiae. Editiones Scientificae. Zagreb.

TILLE, J. M. \& TERRY, R. A. (1963): A two-stage technique for the in vitro digestion of forage crops. J. Br. Grasslandd Soc., 18: 104-111.

TUTIN, et al., (1964-1980): Flora Europaea. Cambridge University Press.

VAN DYNE, G. M. \& TORREL, D. T. (1964): Development and use of the oesophageal fistula: a review. J. Range Management, 17: 7-19.

WILLEMS, J. H. (1985): Growth form spectra and species diversity in permanent grassland plots with different management. En: Sukzession auf Grünlandbrachen. Vorträge eines Symposyums der Arbitsgruppe "Sukzessionsforschung aufDauerflächen" in der Internationalen Vereinigung für Vegetationskunde IV, pp. 35-43. 